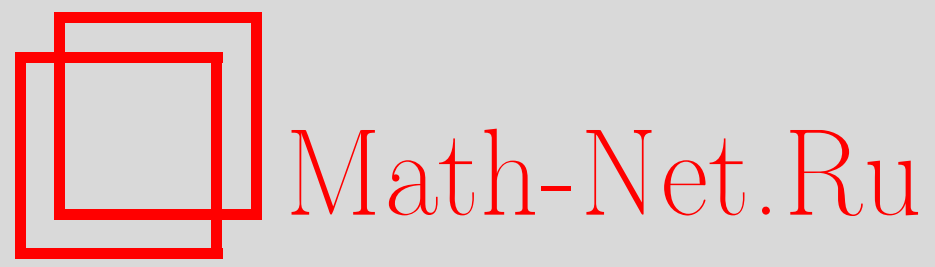

А. И. Аптекарев, Д. Н. Туляков, Четырёхчленные рекуррентные соотношения для $\gamma$-форм, Совр. пробл. матем., 2007, выпуск 9, 37-43

DOI: https://doi.org/10.4213/spm15

Использование Общероссийского математического портала MathNet.Ru подразумевает, что вы прочитали и согласны с пользовательским соглашением

http://www . mathnet.ru/rus/agreement

Параметры загрузки:

IP : 54.166 .219 .16

26 апреля 2023 г., 18:00:00

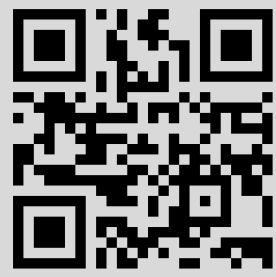




\title{
Четырёхчленные рекуррентные соотношения для $\gamma$-форм ${ }^{1}$
}

\author{
А. И. Аптекарев, Д. Н. Туляков
}

\section{1. Введение}

Пусть $Q_{n}(x)$ - многочлен, задаваемый обобщенной формулой Родрига

$$
Q_{n}(x)=\frac{(1-x)^{-1} e^{x}}{(n !)^{2}} \frac{d^{n}}{d x^{n}} x^{n} \frac{d^{n}}{d x^{n}}(1-x)^{2 n+1} x^{n} e^{-x}
$$

Рассмотрим две математические константы - постоянную Эйлеpa $\gamma$

$$
\gamma:=-\int_{0}^{\infty} \ln x e^{-x} d x
$$

и значение интегральной экспоненты $e \operatorname{Ei}(1,1)$, где

$$
\operatorname{Ei}(n, x)=\int_{1}^{\infty} \frac{e^{-x t}}{t^{n}} d t
$$

Пусть $f_{n}$ и $g_{n}$ - последовательности $\mathbb{Z}$-форм относительно констант $\gamma$ и $e \operatorname{Ei}(1,1)$, генерируемые полиномами $Q_{n}$ по формулам

$$
f_{n}:=p_{n}-\gamma q_{n}:=\int_{0}^{\infty} Q_{n}(x) \ln x e^{-x} d x
$$

и

$$
g_{n}:=e \operatorname{Ei}(1,1) q_{n}-r_{n}:=e \int_{1}^{\infty} Q_{n}(x) \ln x e^{-x} d x .
$$

Настоящая работа посвящена получению и доказательству следующего результата.

\footnotetext{
${ }^{1}$ Работа частично поддержана грантом научных школ НШ-1551.2003.1, программой № 1 ОМН РАН и грантом ИНТАС 03-51-6637. Работа первого автора частично поддержана исследовательским грантом РФФИ-05-0100522, работа второго автора частично поддержана исследовательским грантом РФФИ-05-01-00697.
}

(c) Аптекарев А.И., Туляков Д.Н., 2007 
Теорема 1. Целочисленные коэффициенты $p_{n}, q_{n}, r_{n}$ форм (2) и (3) удовлетворяют рекуррентному соотношению

$$
\begin{aligned}
(16 n & -15) q_{n+1}=\left(128 n^{3}+40 n^{2}-82 n-45\right) q_{n} \\
& -n^{2}\left(256 n^{3}-240 n^{2}+64 n-7\right) q_{n-1}+n^{2}(n-1)^{2}(16 n+1) q_{n-2}
\end{aligned}
$$

с начальными условиями

$$
\begin{aligned}
& p_{0}=0, \quad p_{1}=2, \quad p_{2}=31, \\
& q_{0}=1, \quad q_{1}=3, \quad q_{2}=50, \\
& r_{0}=0, \quad r_{1}=1, \quad r_{2}=24 \text {. }
\end{aligned}
$$

\section{2. Связь $\gamma$-форм с рекуррентными соотношениями для $Q_{n}(1)$}

Известно (см. [1]), что многочлены $Q_{n}^{\left(\alpha_{1}, \alpha_{2}\right)}, \operatorname{deg} Q_{n}^{\left(\alpha_{1}, \alpha_{2}\right)}=4 n$, определяемые формулой Родрига

$$
\begin{aligned}
Q_{n}^{\left(\alpha_{1}, \alpha_{2}\right)}(x)= & \frac{1}{(n !)^{2}}(1-x)^{-1} x^{-\alpha_{2}} e^{x} \\
& \times \frac{d^{n}}{d x^{n}} x^{n+\alpha_{2}-\alpha_{1}} \frac{d^{n}}{d x^{n}}(1-x)^{2 n+1} x^{n+\alpha_{1}} e^{-x},
\end{aligned}
$$

удовлетворяют системе соотношений ортогональности

$$
\left\{\begin{array}{l}
\int_{0}^{1} Q_{n}^{\left(\alpha_{1}, \alpha_{2}\right)}(x) x^{\nu} w_{1}(x) d x=0, \\
\int_{0}^{1} Q_{n}^{\left(\alpha_{1}, \alpha_{2}\right)}(x) x^{\nu} w_{2}(x) d x=0, \\
\int_{1}^{\infty} Q_{n}^{\left(\alpha_{1}, \alpha_{2}\right)}(x) x^{\nu} w_{1}(x) d x=0, \ldots, n-1, \\
\int_{1}^{\infty} Q_{n}^{\left(\alpha_{1}, \alpha_{2}\right)}(x) x^{\nu} w_{2}(x) d x=0,
\end{array}\right.
$$

где $w_{1}(x):=x^{\alpha_{1}}(1-x) e^{-x}, w_{2}(x):=x^{\alpha_{2}}(1-x) e^{-x}$. Нетрудно видеть (вычитая соотношения ортогональности в (6) и деля результат на константу), что соотношения ортогональности (6) сохраняют силу и при

$$
w_{1}(x):=x^{\alpha_{1}}(1-x) e^{-x}, \quad w_{2}(x):=\frac{x^{\alpha_{2}}-x^{\alpha_{1}}}{\alpha_{2}-\alpha_{1}}(1-x) e^{-x} .
$$


Тем самым, устремляя $\alpha_{2} \rightarrow \alpha_{1}$, а затем $\alpha_{1} \rightarrow 0$, мы получаем, что многочлены $Q_{n}:=Q_{n}^{(0,0)}$ (см. (1), (5)) удовлетворяют соотношениям ортогональности (6) с весовыми функциями

$$
w_{1}(x):=(1-x) e^{-x}, \quad w_{2}(x):=(1-x) \ln x e^{-x} .
$$

Рассмотрим следующие функции второго рода, связанные с системой ортогональности (6), (7)

$$
\begin{aligned}
& R_{n}(z)=\int_{0}^{\infty} \frac{Q_{n}(x)}{z-x}(1-x) \ln x e^{-x} d x, \\
& S_{n}(z)=\int_{0}^{1} \frac{Q_{n}(x)}{z-x}(1-x) \ln x e^{-x} d x .
\end{aligned}
$$

Также известно (например, см. [2]), что функции второго рода, порождённые $Q_{n}(x)$, удовлетворяют (вследствие соотношений ортогональности) тем же рекуррентным соотношениям, что и полиномы $Q_{n}(x)$, т.е. рекуррентным соотношениям теоремы $1^{\prime}$ работы [3], и как следствие, рекуррентным соотношениям теорем 1 и 2 работы [4]. Таким образом, интересующие нас формы (2) и (3)

$$
f_{n}=R_{n}(1), \quad g_{n}=e S_{n}(1)
$$

удовлетворяют тому же рекуррентному соотношению, что и $Q_{n}(1)$, например, восьмичленному рекуррентному соотношению следствия теоремы 2 работы [4].

\section{3. Получение четырёхчленного рекуррентного соотношения для форм}

Полученные в [4] рекуррентные соотношения позволили вычислять формы (2) и (3) до $n$ порядка нескольких тысяч, что в свою очередь дало богатый материал для экспериментального изучения рациональных приближений к постоянной Эйлера. В частности, исследуя общие множители последовательностей $\left(a p_{n}+b p_{n-1}+c p_{n-2}\right)$ и $\left(a q_{n}+b q_{n-1}+c q_{n-2}\right)$, был замечен следующий экспериментальный факт:

$\left|\begin{array}{ccc}p_{n} & q_{n} & r_{n} \\ p_{n-1} & q_{n-1} & r_{n-1} \\ p_{n-2} & q_{n-2} & r_{n-2}\end{array}\right| \stackrel{?}{=}(n-1) !^{2}(n-2) !^{2}(16 n-1), \quad n=2,3, \ldots, 5000$ 
что в свою очередь привело к гипотезе, что $p_{n}, q_{n}, r_{n}$ являются базисом решений некоторого четырёхчленного рекуррентного соотношения с рациональными по $n$ коэффициентами. То есть верны соотношения

$$
\left\{\begin{array}{l}
p_{n+1}=K_{n}^{(0)} p_{n}+K_{n}^{(1)} p_{n-1}+K_{n}^{(2)} p_{n-2}, \\
q_{n+1}=K_{n}^{(0)} q_{n}+K_{n}^{(1)} q_{n-1}+K_{n}^{(2)} q_{n-2}, \\
r_{n+1}=K_{n}^{(0)} r_{n}+K_{n}^{(1)} r_{n-1}+K_{n}^{(2)} r_{n-2},
\end{array}\right.
$$

причём коэффициент $K_{n}^{(2)}$ есть отношение последовательных определителей (8).

Экспериментальная проверка гипотезы и получение явного вида рациональных функций $K_{n}^{(0)}, K_{n}^{(1)}$ проводились численной процедурой восстановления рациональной функции по её переопределённому рациональному интерполянту.

\section{4. Доказательство теоремы}

Наша цель - строго обосновать соотношение (4), опираясь на восьмичленное рекуррентное соотношение из работы [4] (следствие теоремы 2). Для того, чтобы не возникало впечатления "везения" или "случайности" в этом обосновании, мы приведём общую процедуру проверки одного линейного рекуррентного соотношения при помощи другого.

Итак, мы находимся в следующей ситуации: есть некоторая последовательность $\left\{S_{n}\right\}_{n=1}^{\infty}$, есть коэффициенты $a_{j}(n)(j=$ $\left.\overline{0, \ldots, m_{1}}\right)$ доказанного соотношения $\sum_{j=0}^{m_{1}} a_{j}(n) S_{n+j}=0$, есть коэффициенты $b_{j}(n)\left(j=\overline{0, \ldots, m_{2}}\right)$ экспериментального соотношения $\sum_{j=0}^{m_{2}} b_{j}(n) S_{n+j} \stackrel{?}{=} 0$, которое требуется проверить и доказать. Нужно узнать, до какого номера $n$ надо проверять экспериментальное соотношение, чтобы быть уверенным в его истинности для всех $n \in \mathbb{N}$. Описанная ниже процедура годится для любых зависимостей коэффициентов $a$ и $b$ от $n$, для которых выполнено следующее свойство:

$(*)$ Любое алгебраическое выражение (полином), составленное из подвыражений вида $a_{j}(n+i), b_{j}(n+i), k$, где $i, j, k \in \mathbb{Z}$, либо обращается в 0 для всех натуральных $n$, либо обращается в 0 на конечном множестве исключительных значений $n \in \mathbb{N}$. 
При этом существует финитная для любого фиксированного выражения процедура проверки альтернативы $(*)$ и нахождения верхней границы на элементы множества исключительных значений. Очевидно, полиномиальная зависимость $a$ и $b$ от $n$ этим свойством обладает.

Будем считать, что $m_{2}<m_{1}$, поскольку элементы $S_{n+j}$ при $j \geqslant m_{1}$ можно выразить через предыдущие. Рассмотрим величины $\Sigma_{n}=\sum_{j=0}^{m_{2}} b_{j}(n) S_{n+j}$. Наша цель - найти рекуррентное соотношение для $\Sigma_{n}$.

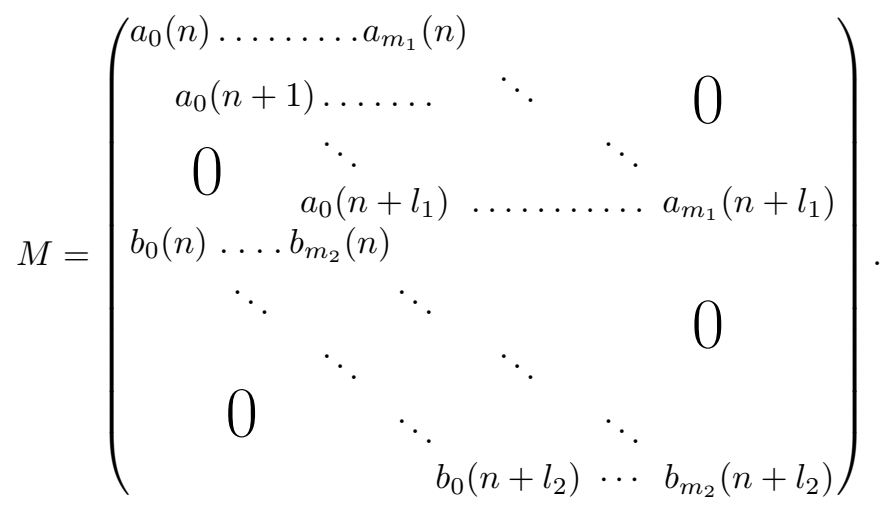

Для этого сформируем матрицу $M$ со структурой, показанной в формуле (9), выбрав $l_{1}$ и $l_{2}$ так, чтобы $l_{1}+m_{1}=l_{2}+m_{2}$ и чтобы $\operatorname{rank}(M)=l_{1}+l_{2}+1$, то есть был на единицу меньше числа строк матрицы $M$. Тогда $0 \leqslant l_{1} \leqslant m_{2}$. Требуемое $l_{1}$ находится следующим образом.

Вначале построим матрицу $M$ для $l_{1}=m_{2} ; l_{2}=m_{1}$. Отметим все столбцы. В матрице, образованной отмеченными столбцами, число строк на 1 больше числа столбцов. Поэтому мы можем сформировать обнуляющий вектор-строку $V$ для отмеченных столбцов: элемент $v_{i}$ получается умножением $(-1)^{i}$ на определитель, полученный из выделенной подматрицы вычёркиванием $i$-й строки. Если последний элемент $V$ не равен 0 (то есть не обращается в 0 при всех $n \in \mathbb{N}$ ), то нужные нам $l_{1}, l_{2}, V$ найдены.

В противном случае можно вычеркнуть последнюю строку, и подматрица из отмеченных столбцов будет вырожденной. Но в последном столбце у неё только один ненулевой элемент $a_{m_{1}}\left(n+l_{1}\right)$. Значит, можно вычеркнуть последний столбец и последние строки в каждом блоке (что равносильно уменьшению $l_{1}$ 
и $l_{2}$ на 1$)$, и всё равно подматрица из выделенных столбцов будет вырожденной. Значит, между отмеченными столбцами есть линейное соотношение, и один из них можно исключить - сделать непомеченым. Последние $l_{2}$ столбцов линейно независимы, поэтому исключаемый столбец можно выбрать среди остальных столбцов. Теперь мы снова находимся в ситуации, когда в матрице, образованной отмеченными столбцами, число строк на 1 больше числа столбцов. Поэтому мы повторяем процедуру формирования обнуляющей строки $V$ для отмеченных столбцов. Поскольку неотмеченные столбцы линейно зависимы от отмеченных, то $V$ является обнуляющей строкой и для них.

Итак, пусть найдены $l_{1}, l_{2}$ и построена обнуляющая строка $V$, которая имеет вид $\left[\varphi_{0}, \ldots, \varphi_{l_{1}}, f_{0}, \ldots, f_{l_{2}}\right]$. По построению $f_{l_{2}} \not \equiv 0$. Рассмотрим матричное произведение $V M W$, где вектор-столбец $W=\left[S_{n}, \ldots, S_{n+m_{1}+l_{1}}\right]^{T}$. Считая его двумя способами, получим тождество:

$$
\begin{aligned}
\sum_{i=0}^{l_{2}} f_{i} \Sigma_{n+i} & =\left[\varphi_{0}, \ldots, \varphi_{l_{1}}, f_{0}, \ldots, f_{l_{2}}\right] \cdot\left[0, \ldots, 0, \Sigma_{n}, \ldots, \Sigma_{n+l_{2}}\right]^{T} \\
& =V \cdot M W=V M \cdot W=[0, \ldots, 0] \cdot W=0 .
\end{aligned}
$$

Поскольку $f_{l_{2}} \not \equiv 0$, то есть значение $n=\tilde{n}$, начиная с которого $f_{l_{2}}(n) \neq 0$. Поэтому проверку $\Sigma_{n} \stackrel{?}{=} 0$ достаточно осуществить до номера $n=\tilde{n}+l_{2}$.

Применим эту процедуру к рекуррентным соотношениям (4) и (16) из работы [4]. Хотя коэффициенты рекуррентного восьмичленного соотношения являются многочленами (по $n$ ) очень большой степени (порядка 80-90), тем не менее современные пакеты символьных вычислений (MAPLE) позволяют вычислять определители из (9) с такими входными данными. Для соотношения (4) и восьмичленного соотношения (16) из работы [4] оказалось, что ранг матрицы (9) для $l_{1}=m_{2}=3$ и $l_{2}=m_{1}=7$ равен $8=\left(l_{1}+l_{2}+1\right)-3$, поэтому этап уменьшения $l_{1}, l_{2}$ будет пройден 3 раза. В итоге $l_{1}=0, l_{2}=4$, отмечать можно последние 5 столбцов, и вычисленная по ним аннулирующая строка $V$ аннулирует и первые 3 столбца. Вычисленный таким образом многочлен $f_{4}(n)$ 
(последний элемент $V$ ) имеет вид

$$
f=f_{4}(n)=\left|\begin{array}{ccccc}
a_{3}(n) & a_{4}(n) & a_{5}(n) & a_{6}(n) & a_{7}(n) \\
b_{3}(n) & 0 & 0 & 0 & 0 \\
b_{2}(n+1) & b_{3}(n+1) & 0 & 0 & 0 \\
b_{1}(n+2) & b_{2}(n+2) & b_{3}(n+2) & 0 & 0 \\
b_{0}(n+3) & b_{1}(n+3) & b_{2}(n+3) & b_{3}(n+3) & 0
\end{array}\right|
$$

Оценку величины максимального по модулю корня этого полинома делаем через коэффициенты полинома по известной формуле:

$$
|\tilde{n}|<1+\max _{1 \leqslant i \leqslant \operatorname{deg}(f)}\left|\frac{\operatorname{coeff}(f, \operatorname{deg}(f)-i)}{\operatorname{coeff}(f, \operatorname{deg}(f))}\right|^{1 / i} .
$$

Подставляя сюда явные значения коэффициентов рекуррентных соотношений (4) и (16) из работы [4], получаем

$$
|\tilde{n}|<70 .
$$

Численная проверка совпадения решений рекуррентных соотношений (4) и (16) из [4] до $n=70$ завершает доказательство теоремы.

\section{Список литературы}

[1] A. I. Aptekarev, A. Branquinho, W. Van Assche, "Multiple orthogonal polynomials for classical weights", Trans. Amer. Math. Soc., 355:10 (2003), 3887-3914 doi 10.1090/S0002-9947-03-03330-0, MR 1990569, Zbl 1033.33002.

[2] В. А. Калягин, "Аппроксимации Эрмита-Паде и спектральный анализ несимметричных операторов", Матем. сб., 185:6 (1994), 79-100 Mi sm903, MR 1280397, Zbl 0840.47026.

[3] Д. В. Христофоров, "Рекуррентные соотношения для аппроксимаций Эрмита-Паде одной системы из четырех функций марковского и стилтьесовского типа", Современные проблемы математики, вып. 9, ред. А. И. Аптекарев, МИАН, М., 2006, 11-26.

[4] А. И. Боголюбский, "Рекуррентные соотношения с рациональными коэффициентами для некоторых совместно ортогональных многочленов, задаваемых формулой Родрига", Современные проблемы математики, вып. 9, ред. А. И. Аптекарев, МИАН, М., 2006, 27-35. 